\title{
ASYMPTOTIC BEHAVIOURS OF A CLASS OF INTEGRAL TRANSFORMS IN COMPLEX DOMAINS
}

\author{
by R. S. PATHAK AND S. K. MISHRA
}

(Received 28th October 1987)

\section{Introduction}

Zemanian [17] obtained abelian theorems for the Hankel and $K$-transforms of functions and then extended his results to the corresponding transforms of distributions in the sense of Schwartz [11]. Jones [6] has discussed at length asymptotic behaviours of transforms generalized in his sense. Following the technique of Zemanian many authors have obtained abelian theorems for more general transforms of functions and distributions in the sense of Schwartz. Mention may be made of the works of Joshi and Saxena [7], Lavoine and Misra [8] and Pathak [10]. However, these authors were confined to the transforms of real variables only.

Carmichael and Milton [2] obtained abelian theorems for the distributional Stieltjes transform when the variable $s$ of the transform was complex. They allowed $|s|$ to tend to zero or to infinity staying in the wedge domain $\left\{s:\left|s_{2}\right| \leqq K s_{1} ; s=s_{1}+i s_{2}, s_{1} \geqq 0\right\}$. They also used Lojasiewicz's definition of limits of a distribution and thus extended the work of Lavoine and Misra [8]. Takači [13] applied the idea of quasi-asymptotic behaviour of distributions due to Drožžinov and Zav'jalov [4] and extended the work of Lavoine and Misra based on the notion of equivalence at infinity. Some general results on asymptotic behaviours of $H$-transforms using quasi-asymptotic behaviours have recently been obtained by Carmichael and Pathak [3].

There exists a class of integral transforms in which integration is performed with respect to a parameter (sometimes order of the kernel) instead of the argument-variable. The well-known examples are Kontorovich-Lebedev and Mehler-Fock transforms and their generalization due to Wimp [15]. Asymptotic behaviours of all these transforms have not been much discussed. The work of Wong [16] on asymptotic expansion of the Kontorovich-Lebedev transform is worth a mention here. The aim of the present paper is to investigate asymptotic behaviours of a generalization of the Wimp transform which incorporates all the aforesaid and some other transforms as its special cases [15].

In this paper we define the generalized Wimp transform ( $W$-transform) of functions in the complex domain, and obtain initial and final value theorems for the transform when the transform variable $s \in \mathbb{C}$ approaches zero (or infinity) in a wedge. We define the $W$ transform of a large class of distributions. Following Stankovic [12] we introduce the 
idea of quasi-asymptotic behaviours (q.a.b.) of our $W$-transformable generalized functions and obtain initial and final value theorems for the $W$-transform of distributions.

\section{The $G$-Function}

The Meijer's $G$-function is defined by the Mellin-Barnes contour integral

$$
G_{p, q}^{m, n}\left(\left.z\right|_{b_{1}, \ldots, b q} ^{a_{1}, \ldots, a_{p}}\right)=G_{p, q}^{m, n}\left(\left.z\right|_{b_{q}} ^{a_{p}}\right)=\frac{1}{2 \pi i} \int_{L} \frac{\prod_{j=1}^{m} \Gamma\left(b_{j}-s\right) \prod_{j=1}^{n} \Gamma\left(1-a_{j}+s\right)}{\prod_{j=m+1}^{q} \Gamma\left(1-b_{j}+s\right) \prod_{j=n+1}^{p} \Gamma\left(a_{j}-s\right)}-z^{s} d s
$$

where the empty product is interpreted as unity. In (2.1) $0 \leqq m \leqq q, 0 \leqq n \leqq p$ and the path $L$ runs from $-i \infty$ to $i \infty$ so that the poles of $\Gamma\left(b_{j}-s\right), j=1, \ldots, m$, are to the right and all the poles of $\Gamma\left(1-a_{j}-s\right), j=1, \ldots, n$ are to the left of $L$. Here $a_{j}, b_{j}$ and $z(\neq 0)$ are complex numbers. For convergence of the integral in (2.1) we need $p+q<2(m+n)$ and $|\arg z|<\left(m+n-\frac{1}{2} p-\frac{1}{2} q\right) \pi$. For various properties of the $G$-function refer to [9].

The kernel of our transformation is

$$
G(z, s):=G_{p+2, q}^{m, n+2}\left(\left.z\right|_{b_{q}} ^{1-v+s, 1-v-s, a_{p}}\right)
$$

where $z=z_{1}+i z_{2}, v=v_{1}+i v_{2}$ and $s=s_{1}+i s_{2}$ are complex numbers. We shall use the following notation:

$$
\begin{aligned}
& \rho=m+n+1-\frac{1}{2}(p+q), \quad \sigma=q-m-n-2 \\
& \mu=\sum_{j=1}^{q} b_{j}-\sum_{j=1}^{p} a_{j} \\
& c=\min \operatorname{Re} b_{j}, \quad j=1,2, \ldots, m \\
& d=\max \left(\operatorname{Re} a_{j}-1,-v_{1}\right), \quad j=1,2, \ldots, n \\
& b=\max (d,-1) .
\end{aligned}
$$

Certain restrictions on the parameters of the $G$-function are required for the validity of our results. These are called (A) and (B), see [9, p. 178].

Let $1 \leqq p+2<q$.

(A) $\quad 1-v \pm s-b_{h} \neq 1,2,3, \ldots, a_{j}-b_{h} \neq 1,2,3, \ldots$ for $j=1,2, \ldots, p ; h=1,2, \ldots, q$.

(B) $\quad 1-v \pm s-a_{r} \neq 0, \pm 1, \pm 2, \ldots ; a_{j}-a_{r} \neq 0, \pm 1, \pm 2, \ldots$ for $j=1,2 \ldots, p ; h=$ $1,2, \ldots, q ; s \neq 0, \pm 1, \pm 2$. 
Lemma 2.1. Let

(1) $s=s_{1}+i s_{2}$ be an element of the strip $\Omega=\left\{s \in \mathbb{C}:-v_{1}-c<s_{1}<v_{1}+\min (c, d)\right\}$;

(2) $0 \leqq m \leqq q, 0 \leqq n \leqq p, p+q \leqq 2(m+n+1), p+2<q$;

(3) $|\arg z| \leqq \rho \pi,|\arg s| \leqq \pi$.

Then

$$
|G(z, s)| \leqq A|z|^{c}\left|\frac{\Gamma\left(v+b_{h}+s\right) \Gamma\left(v+b_{h}-s\right)}{\Gamma\left(2 v+2 b_{h}\right)}\right|, \quad \text { for }|z|<1,
$$

where $c=\operatorname{Re} b_{h}=\min _{1 \leq j \leq m} \operatorname{Re} b_{j}$, and $A$ is independent of $z$ and s; also

$$
|G(z, s)| \leqq B|z|^{d}\left|\frac{\Gamma\left(v+a_{k}+s-1\right) \Gamma\left(v+a_{k}-s-1\right)}{\Gamma\left(2 v+2 a_{k}-2\right)}\right|, \quad \text { for }|z|>1,
$$

where

$$
d+1=\operatorname{Re} a_{k}=\max _{0 \leqq j \leqq n} \operatorname{Re} a_{j} \text { with } a_{0}=-v_{1}+1 .
$$

Proof. From Wimp [15, Theorem 2, pp. 34-35] we have

$$
\begin{aligned}
& \frac{2^{2 v-1}}{\sqrt{\pi}} \int_{0}^{\infty} x^{s-1}\left(\frac{x}{(1+x)^{2}}\right)^{v} G_{p+2, q}^{m, n+2}\left(\left.\frac{4 z x}{(1+x)^{2}}\right|_{b_{q}} ^{1-v,(1 / 2)-v, a_{p}}\right) d x \\
& =G_{p+2, q}^{m, n+2}\left(\left.z\right|_{b_{q}} ^{1-v+s, 1-v-s, a_{p}}\right):=G(z, s)
\end{aligned}
$$

valid under the conditions (1)-(3) of the lemma.

Now, replacing the $G$-function within the integral sign (2.6) by its series representation [9, (7), p. 145] for $|z|<1$ and by the asymptotic expansion [9, (1), p. 190] for $|z|>1$ one can derive the estimates (2.4) and (2.5) respectively.

For $K \geqq 0$, a fixed real number, we define a wedge $Q_{K}$ in the upper half plane $\mathbb{C}^{U}$ by

$$
Q_{K}:=\left\{s: s=s_{1}+i s_{2} \in \Omega \subset \mathbb{C} \text { and }\left|s_{1}\right| \leqq \mathrm{Ks}_{2}\right\} .
$$

Lemma 2.2 For complex $v, \beta, \eta$ and $s \in Q_{K},|s| \rightarrow \infty$,

$$
\left|\frac{\Gamma(v+\beta+s) \Gamma(v+\beta-s)}{\Gamma(v-\eta+s) \Gamma(v-\eta-s)}\right| \leqq C\left(1+K^{2}\right)^{\left(\beta_{1}+\eta_{1}\right)} s_{2}^{2\left(\beta_{1}+\eta_{1}\right)} \exp \left(2 \pi\left(\beta_{2}+\eta_{2}\right)\right),
$$

where

$$
C>0, \beta=\beta_{1}+i \beta_{2}, \eta=\eta_{1}+i \eta_{2} .
$$

Proof. Using the formula $[9,2.2(2), p .11]$ we have 


$$
\frac{\Gamma(v+\beta+s) \Gamma(v+\beta-s)}{\Gamma(v-\eta+s) \Gamma(v-\eta-s)}=\frac{\Gamma(v+\beta+s)}{\Gamma(v-\eta+s)} \frac{\Gamma(1-v+\eta+s)}{\Gamma(1-v-\beta+s)} \frac{\sin (v-\eta-s) \pi}{\sin (v+\beta-s) \pi}
$$

Next an application of the formula $[9,(11)$, p. 33] gives

$$
\left|\frac{\Gamma(v+\beta+s) \Gamma(v+\beta-s)}{\Gamma(v-\eta+s) \Gamma(v-\eta-s)}\right| \leqq C\left|s^{2(\eta+\beta)}\right| \text { for }|\arg s| \leqq \pi-\varepsilon, \varepsilon>0 .
$$

For $s \in Q_{K}$ this yields the estimate (2.7).

In the study of the distributional generalized Wimp transform we shall need the following differentiation formula [9, p. 152].

$$
z^{r}\left(\frac{d}{d z}\right)^{r} G_{p, q}^{m, n}\left(\left.z\right|_{b_{q}} ^{a_{p}}\right)=G_{p+1, q+1}^{m, n+1}\left(\left.z\right|_{b_{q}, r} ^{0, a_{p}}\right)
$$

The following integral is well-known [9, p. 157]:

$$
\int_{0}^{\infty} t^{\eta} G(t, s) d t=\Gamma(v-s-\eta-1) \Gamma(v+s-\eta-1) \mathscr{H}(\eta)
$$

where

$$
\mathscr{H}(\eta)=\frac{\prod_{j=1}^{m} \Gamma\left(b_{j}+\eta+1\right) \prod_{j=1}^{n} \Gamma\left(-a_{j}-\eta\right)}{\prod_{j=m+1}^{q} \Gamma\left(-b_{j}-\eta\right) \prod_{j=n+1}^{p} \Gamma\left(a_{j}+\eta+1\right)}
$$

$1 \leqq n+2 \leqq p+2<q, \quad 1 \leqq m \leqq q, \quad p+q \leqq 2(m+n+1), \quad-c-1<\operatorname{Re} \eta<-\max _{1 \leqq j \leqq n}\left(a_{j}\right)$, and $\left|s_{1}\right|<v_{1}-\eta_{1}-1$.

\section{The generalized Wimp transform of functions}

Let $s=s_{1}+i s_{2}$ be a complex variable. Then the generalized Wimp transform $F(s)$ of a complex valued function $f(t), 0 \leqq t<\infty$, is defined by

$$
F(s)=\int_{0}^{\infty} G(t, s) f(t) d t
$$

whenever the integral (3.1) is convergent. The transform for $s=i x$ was considered by Wimp [15]. As far as is known to the author the asymptotic behaviours of the transform have not been investigated. In what follows we shall obtain abelian theorems 
for (3.1) by relating the behaviour of $f(t)$ when $t \rightarrow 0+$ (infinity) to the behaviour of the transform $F(s)$ when $|s| \rightarrow \infty$ staying in the wedge $Q_{K}$. We shall assume that the complex variable $s \in \mathbb{C}^{U}=\left\{s: s=s_{1}+i s_{2} \in \mathbb{C}, s_{2}>0\right\}$.

Our initial value theorem for (3.1) is:

Theorem 3.1. Let $\eta=\eta_{1}+i \eta_{2}$ be a complex number with $-1<c+\eta_{1}<0, d+\eta_{1}<-1$. Let $f(t), 0 \leqq t<\infty$, be a complex valued function such that $\left(t^{d} f(t)\right)$ is absolutely integrable over $(y, \infty)$ for all $y>0$ and such that $\left(t^{-\eta} f(t)\right)$ is bounded on $0<t \leqq y$ for all $y>0$. Let the generalized Wimp transform $F(s)$ of $f(t)$ exist for all $s \in \mathbb{C}^{U}$. If there is a complex number $\Lambda$ for which

$$
\lim _{t \rightarrow 0+} t^{-\eta} f(t)=\Lambda
$$

then for each fixed $K \geqq 0$ with $Q_{K} \subset \mathbb{C}^{U} \cap\left\{s:\left|s_{1}\right|<v_{1}-\eta_{1}-1\right\}$,

$$
\lim _{\substack{|s| \rightarrow \infty \\ s \in Q_{K}}}(\Gamma(v-s-\eta) \Gamma(v+s-\eta) \mathscr{H}(\eta))^{-1} F(s)=\Lambda
$$

where $\mathscr{H}(\eta)$ is defined by (2.9).

Proof. From (3.1) and (2.9) we have

$$
\begin{aligned}
& (\Gamma(v-s-\eta) \Gamma(v+s-\eta) \mathscr{H}(\eta))^{-1} F(s)-\Lambda \\
& \quad=(\Gamma(v-s-\eta) \Gamma(v+s-\eta) \mathscr{H}(\eta))^{-1} \int_{0}^{\infty} t^{\eta} G(t, s)\left(t^{-\eta} f(t)-\Lambda\right) d t \\
& \quad=I_{1}+I_{2} \text { (say), where for fixed } y>0, \\
& I_{1}=(\Gamma(v-s-\eta) \Gamma(v+s-\eta) \mathscr{H}(\eta))^{-1} \int_{0}^{y} t^{\eta} G(t, s)\left(t^{-\eta} f(t)-\Lambda\right) d t
\end{aligned}
$$

and

$$
I_{2}=(\Gamma(v-s-\eta) \Gamma(v+s-\eta) \mathscr{H}(\eta))^{-1} \int_{y}^{\infty} t^{\eta} G(t, s)\left(t^{-\eta} f(t)-\Lambda\right) d t
$$

Since $|s| \rightarrow \infty$, we can use the estimate (2.4) and apply Lemma 2.2 to get

$$
\left|I_{1}\right| \leqq\left|(\Gamma(v-s-\eta) \Gamma(v+s-\eta) \mathscr{H}(\eta))^{-1}\right| A\left|\Gamma\left(v+b_{h}+s\right) \Gamma\left(v+b_{h}-s\right)\right| \int_{0}^{y} t^{c+\eta_{1}}\left|t^{-\eta} f(t)-\Lambda\right| d t
$$




$$
\leqq A_{1} s_{2}^{2\left(c+\eta_{1}\right)} \int_{0}^{y} t^{c+\eta_{1}}\left|t^{-\eta} f(t)-\Lambda\right| d t
$$

where $A_{1}$ is an appropriate positive constant. Thus

$$
\left|I_{1}\right| \leqq A_{1} s_{2}^{2\left(c+\eta_{1}\right)} \sup _{0<t<y}\left|t^{-\eta} f(t)-\Lambda\right| y^{c+\eta_{1}+1} /\left(c+\eta_{1}+1\right) .
$$

Now, choose $-1<c+\eta_{1} \leqq 0$. Then for given $\varepsilon>0$ we can choose $y$ small enough such that $\left|I_{1}\right|<\varepsilon / 2$; fix this $y$.

Next we use the estimate (2.5) and apply Lemma 2.2 to $I_{2}$. Then

$$
\begin{aligned}
\left|I_{2}\right| \leqq & \left|(\Gamma(v-s-\eta) \Gamma(v+s-\eta) \mathscr{H}(\eta))^{-1}\right| \\
& \times B\left|\Gamma\left(v+a_{k}+s-1\right)\right|\left|\Gamma\left(v+a_{k}-s-1\right)\right| \int_{y}^{\infty} t^{d+\eta_{1}}\left|t^{\eta} f(t)-\Lambda\right| d t \\
& \leqq B_{1} s_{2}^{2\left(d+\eta_{1}\right)} \int_{y}^{\infty} t^{d}\left|f(t)-\Lambda t^{\eta_{1}}\right| d t .
\end{aligned}
$$

For $d+\eta_{1}+1<0$, and fixed $y>0$ as in $I_{1}$, the last integral is absolutely convergent due to the hypothesis on $f(t)$. Hence $I_{2}$ can be made less than $\varepsilon / 2$ by choosing $s_{2}$ large enough. This proves the theorem.

A final value theorem for the transform (3.1) is the following:

Theorem 3.2. Let $\eta=\eta_{1}+i \eta_{2}$ be complex with $-1<c+\eta_{1}<0, d+\eta_{1}+1<0$. Let $f(t)$ be a complex valued function on $0 \leqq t<\infty$, such that $\left(t^{c} f(t)\right)$ is absolutely integrable over $(0, y)$ for all $y>0$, and such that $\left(t^{-n} f(t)\right)$ is bounded on $y \leqq t<\infty$ for all $y>0$. Let the generalized Wimp transform $F(s)$ of $f(t)$ exist for all $s \in \mathbb{C}^{U}$. If there exists a complex number $\Lambda$ for which

$$
\lim _{t \rightarrow \infty} t^{-\eta} f(t)=\Lambda,
$$

then for each fixed $K \geqq 0$ with $Q_{K} \subset \mathbb{C}^{U} \cap\left\{s:\left|s_{1}\right|<v_{1}-\eta_{1}-1\right\}$,

$$
\lim _{\substack{|s| \rightarrow \infty \\ s \in Q_{\mathbf{K}}}}(\Gamma(v-s-\eta) \Gamma(v+s-\eta) \mathscr{H}(\eta))^{-1} F(s)=\Lambda
$$

where $\mathscr{H}(\eta)$ is defined by (2.9).

Proof. Proceeding as in the proof of Theorem 3.1 we have 


$$
\left|(\Gamma(v-s-\eta) \Gamma(v+s-\eta) \mathscr{H}(\eta))^{-1} F(s)-\Lambda\right| \leqq I_{1}+I_{2} .
$$

Since $|s| \rightarrow \infty$, using the boundedness hypothesis on $\left(t^{-\eta} f(t)\right)$ we can write

$$
\left|I_{2}\right| \leqq B_{1} s_{2}^{2\left(d+\eta_{1}\right)} \sup _{i>y}\left|t^{-\eta} f(t)-\Lambda\right| \int_{y}^{\infty} t^{d+\eta_{1}} d t
$$

Let $d+\eta_{1}+1<0$. Then, for given $\varepsilon>0$, we can choose $y$ large enough to make $\left|I_{2}\right|<\varepsilon / 2$; so fix $y$. Also,

$$
\left|I_{1}\right| \leqq A_{1} s_{2}^{2\left(c+\eta_{1}\right)} \int_{0}^{y} t^{c}\left|f(t)-\Lambda t^{\eta_{1}}\right| d t .
$$

For $-1<c+\eta_{1}<0$, and fixed $y>0$ as in $I_{2}$, the last integral is absolutely convergent due to the hypothesis on $f(t)$. Hence $\left|I_{1}\right|$ can be made less then $\varepsilon / 2$ by choosing $s_{2}$ large enough. This, coupled with the conclusion concerning $I_{1}$, completes the proof of Theorem 3.2.

\section{The generalized Wimp transform of distributions}

Let $b$ and $c$ be defined by (2.3). Then Zemanian's test function space $M_{c, b}$ consists of all complex valued $C^{\infty}$-functions $\phi$ on $I=(0, \infty)$ which satisfy

$$
\xi_{k}(\phi)=\sup _{0<t<\infty}\left|\zeta_{c, b}(t) t^{k+1} D_{t}^{k} \phi(t)\right|<\infty,
$$

where $\xi_{c, b}$ is a smooth function such that

$$
\zeta_{c, b}(t)= \begin{cases}t^{-c} & 0<t \leq 1 \\ t^{-b} & 2 \leq t<\infty\end{cases}
$$

The topology of $M_{c, b}$ is generated by $\left\{\xi_{k}\right\}_{k=0}^{\infty} . M_{c, b}$ is a complete countably multinormed space. Its dual is denoted by $M_{c, b}^{\prime}$. For its various properties we refer to [18].

Lemma 4.1. For $s \in \Omega^{*}:=\left\{s \in \mathbb{C},-v_{1}-c<s_{1}<v_{1}+\min (c, b)\right\}, G(t, s) \in M_{c+1, b+1}$ as a function of $t>0$.

Proof. Using (2.8) we have

$$
\begin{aligned}
\left|J_{k}(t)\right| & \equiv\left|\zeta_{c+1, b+1}(t) t^{k+1} D_{t}^{k} G(t, s)\right| \\
& =\left|\zeta_{c+1, b+1}(t) t G_{p+3, q+1}^{m, n+3}\left(\left.t\right|_{b_{q, k}, 1-v+s, 1-v-s, a_{p}} ^{0,1}\right)\right|
\end{aligned}
$$

Using analogues of the estimates (2.4) and (2.5) we have 


$$
\begin{array}{cc}
\left|J_{k}(t)\right| \leqq A \mid t^{-c} t^{c} \frac{\Gamma\left(v+b_{h}+s\right) \Gamma\left(v+b_{h}-s\right)}{\Gamma\left(2 v+2 b_{h}\right)} & \text { for } 0<t \leqq 1 \\
\leqq B \mid t^{-b} t^{b} \frac{\Gamma\left(2 v+a_{l}+s-1\right) \Gamma\left(v+a_{l}-s-1\right)}{\Gamma\left(2 v+2 a_{l}-2\right)} & \text { for } 1<t<\infty
\end{array}
$$

where $b=\max (d,-1)$. Therefore $\left|J_{k}(t)\right|$ is bunded uniformly for all $t$ by a constant independent of $t$ for each $k=0,1,2, \ldots$.

In view of Lemma 4.1 we can define the generalized Wimp transform of $v \in M_{c+1, b+1}^{\prime}$ by

$$
F[v ; s]=\langle v(t), G(t, s)\rangle, \quad s \in \Omega^{*}
$$

Lemma 4.2. Let $v \in M_{c+1, b+1}^{\prime} . F[v ; s]$ is analytic for $s \in Q_{k} \cap \Omega^{*}$ and there is a constant $P$ which is independent of $s \in Q_{k}$ such that

$$
\begin{aligned}
|F[v ; s]| & \leqq P \quad \text { for } 0<|s| \leqq 1 \\
& \leqq P e^{-s_{2} \pi} s_{2}^{2 v_{1}+2 \max (c, b)} \quad \text { for } 1<|s|<\infty
\end{aligned}
$$

Proof. The proof of analyticity can be given in the usual way [18, p. 58]. For the growth we apply [18, Theorem 1.8-1, p. 18] and get a constant $K$ and a non-negative integer $N$ such that

$$
\begin{aligned}
|F[v ; s]| & \leqq K \max _{0 \leqq k \leqq N} \sup _{0<t<\infty}\left|\zeta_{c+1, b+1}(t) t^{k+1} D_{t}^{k} G(t, s)\right| \\
& \leqq K \max _{k} \sup _{t}\left|\zeta_{c+1, b+1}(t) t G_{p+3, q+1}^{m, n+3}\left(\left.t\right|_{b_{q, k}, \mathbf{1}, v+s, 1-v-s, a_{p}} ^{0,1}\right)\right| \\
& \leqq A_{1}\left|\Gamma\left(v+b_{h}+s\right) \Gamma\left(v+b_{h}-s\right)\right|+B_{1}\left|\Gamma\left(v+a_{l}+s-1\right) \Gamma\left(v+a_{l}-s-1\right)\right|
\end{aligned}
$$

on using inequalities (4.3) and (4.4). Here $A_{1}$ and $B_{1}$ are positive constants independent of $s$. So that for $|s| \rightarrow 0+, F[v ; s]$ is bounded by a constant independent of $s$.

Further, for large $s \in Q_{k} \cap \Omega^{*}$, proceeding as in Lemma 2.2 we can show that

$$
|F[v ; s]| \leqq A_{2} s_{2}^{2\left(v_{1}+c\right)} e^{-s_{2} \pi}+B_{2} s_{2}^{2\left(v_{1}+b\right)} e^{-s_{2} \pi}
$$

from which we get the required inequality.

\section{Quasi-asymptotics and abelian theorems}

In this section we define quasi-asymptotic behaviour (q.a.b.) of generalized functions 
in $M_{c, b}^{\prime}$ and obtain abelian theorems for the $W$-transform of generalized functions in $M_{c+1, b+1}^{\prime}$ possessing q.a.b. By $M_{c, b}^{\prime}(\widetilde{I})$ we mean those generalized functions in $M_{c, b}^{\prime}$ which have support in $\bar{I}=[0, \infty)$.

According to Bojanic and Karamata [1], a function $L(x)$ is a slowly varying function if $L$ is real valued, positive for $x>0$ and measurable on every finite interval $[a, b]$, $0<a<b<\infty$, and if

$$
\lim _{t \rightarrow \infty} L(u t) / L(t)=1 \quad \text { for each } u>0 .
$$

In $[1$, pp. $3-4]$ it is proved that for any $\eta>0$

$$
\begin{gathered}
x^{\eta} L(x) \rightarrow \infty \text { and } x^{-\eta} L(x) \rightarrow 0(x \rightarrow \infty) ; \\
\sup _{0 \leqq t \leqq x}\left\{t^{\eta} L(t)\right\} \simeq x^{\eta} L(x)(x \rightarrow \infty) \\
\sup _{t \geqq x}\left\{t^{-\eta} L(t)\right\} \simeq x^{-\eta} L(x)(x \rightarrow \infty) .
\end{gathered}
$$

Definition 5.1. A generalized function $T \in M_{c, b}^{\prime}(\bar{I})$ has q.a.b. at infinity of order $\alpha$ if

$$
\lim _{k \rightarrow \infty}\left(k^{\alpha} L(k)\right)^{-1} T(k t):=\gamma(t) \quad \text { in } \quad M_{c, b}^{\prime}
$$

and $T$ has q.a.b. at zero of order $\alpha$ if

$$
\lim _{k \rightarrow 0+}\left(k^{\alpha} L(1 / k)\right)^{-1} T(k t):=\gamma(t) \quad \text { in } \quad M_{c, b}^{\prime}
$$

provided $\gamma(t) \neq 0$. Then we write

$$
T(t) \sim \gamma(t)
$$

in $M_{c, b}^{\prime}$ when $t \rightarrow \infty(t \rightarrow 0+$ resp.). If for any $\alpha$

$$
\lim _{k \rightarrow \infty}\left(k^{\alpha} L(k)\right)^{-1} T(k t)=0 \text { in } M_{c, b}^{\prime}
$$

or,

$$
\lim _{k \rightarrow 0+}\left(k^{\alpha} L(1 / k)\right)^{-1} T(k t)=0 \quad \text { in } M_{c, b}^{\prime}
$$

we say that $T$ has order $-\infty$ as $t \rightarrow \infty(t \rightarrow 0+$ resp.) and write

$$
T \sim 0
$$


in $M_{c, b}^{\prime}$ as $t \rightarrow \infty(0+)$.

Following the method of Drožžinov and Zav'jalov [4, p. 330] it can be shown that $\gamma$ is a homogeneous generalized function of order $\alpha$. Using the arguments of Gel'fand and Shilov [5, p. 80] it follows that

$$
\gamma(t)=c f_{\alpha+1}(t), \quad t>0,
$$

where

$$
\begin{gathered}
f_{\alpha+1}(t)=I(t) t^{\alpha} / \Gamma(\alpha+1) \text { for } \alpha>0 \\
f_{\alpha+1}(t)=D^{n} f_{\alpha+n+1}(t) \text { for } \alpha \leqq 0 \text { and } \alpha+n>0 .
\end{gathered}
$$

Here $I(t)$ is the characteristic function of $[0, \infty)$.

Using a standard method [14, p. 273] the following representation of elements of $M_{c, b}^{\prime}$ can be given. A generalized function $T \in M_{c, b}^{\prime}$ is of the form

$$
T=\sum_{r=0}^{N}(-1)^{r} D_{r}^{r}\left(\zeta_{c, b}(t) t^{r+1} D_{t} h_{r}(t)\right)
$$

for some non-negative integer $N$, where $h_{r} \in L^{\infty}(I), r=0,1, \ldots, N$.

The following theorem gives a characterization of the elements of $M_{c+1, b+1}^{\prime}(\bar{I})$ possessing q.a.b.

Theorem 5.2 Let $T$ be a generalized function belonging to $M_{c+1, b+1}^{\prime}(\bar{I})$ such that for some positive integer $N$

$$
T=D_{t}^{N}\left(\zeta_{c+1, b+1}(t) t^{N+1} G(t)\right)
$$

$G(t)$ is a continuous function having support in $[0, \infty)$ and $c \leqq 0$. If $T$ has q.a.b. at infinity of order $\alpha$, then there exists a complex constant $Q$ such that

$$
\lim _{t \rightarrow \infty}\left(t^{\alpha-1} L(t)\right)^{-1} \zeta_{c+1, b+1}(t) G(t)=Q
$$

Also, if $T$ has q.a.b. at zero of order $\alpha$, then there exists a complex constant $Q$ such that

$$
\lim _{t \rightarrow 0+}\left(t^{\alpha-1} L(1 / t)\right)^{-1} \zeta_{c+1, b+1}(t) G(t)=Q
$$

Conversely, if $\alpha+c+\eta>0, \eta<1 ; \alpha+b+\eta^{\prime}<0, \eta^{\prime}>1$ and (5.10) holds then $T$ has q.a.b. at infinity of order $\alpha$. And if $\alpha+c+\eta>0, \eta<1 ; \alpha+b+\eta^{\prime}<0, \eta^{\prime}>1,(5.11)$ holds and $\int_{1}^{\infty}|G(t)| d t<\infty$, then $T$ has q.a.b. at zero of order $\alpha$.

Proof. Let $T \in M_{c+1, b+1}^{\prime}(I)$ be of the form (5.9) and have q.a.b. at infinity of order $\alpha$. Then by Definition 5.1 there is an element $\gamma \in M_{c+1, b+1}^{\prime}$ such that 


$$
\lim _{k \rightarrow \infty}\left\langle\left(k^{\alpha} L(k)\right)^{-1} T(k t), \phi(t)\right\rangle=\langle\gamma, \phi\rangle, \phi \in M_{c+1, b+1},
$$

where $\langle\gamma, \phi\rangle$ is a complex number. Choosing $\phi(t)=\left(I(1-t)(1-t)^{N-1}\right) / \Gamma(N)$, we have $\phi(t) \in M_{c+1, b+1}^{(m)}=\left\{\phi \in C^{m}(I) / \xi_{k}(\phi)<\infty, k=0,1, \ldots, m\right\}$ for $m<N-1$ and $c \leqq 0$. Using (5.7) we have

$$
\begin{aligned}
& \left(k^{\alpha} L(k)\right)^{-1}\left\langle T(k t),\left(I(1-t)(1-t)^{N-1}\right) / \Gamma(N)\right\rangle \\
& \quad=k^{-\alpha-N}(L(k))^{-1}\left\langle T(u),\left(I(1-u / k)(k-u)^{N-1}\right) / \Gamma(N)\right\rangle \\
& \quad=k^{-\alpha-N}\left\langle(L(k))^{-1}\left\langle D_{u}^{N}\left(\zeta_{c+1, b+1}(u) u^{N+1} G(u)\right),\left(I(k-u)(k-u)^{N-1}\right) / \Gamma(N)\right\rangle\right. \\
& \quad=k^{-\alpha-N}(L(k))^{-1} \int_{0}^{k} D_{u}\left(\zeta_{c+1, b+1}(u) u^{N+1} G(u)\right) d u \\
& \quad=\left(k^{\alpha-1} L(k)\right)^{-1} \zeta_{c+1, b+1}(k) G(k) .
\end{aligned}
$$

Thus $\left(k^{a-1} L(k)\right)^{-1} \zeta_{c+1, b+1}(k) G(k)$ tends to

$$
Q=\left\langle\gamma(t),\left(I(1-t)(1-t)^{N-1}\right) / \Gamma(N)\right\rangle \quad \text { as } \quad k \rightarrow \infty
$$

because of (5.12) and the choice of $\phi(t)$ above; this proves (5.10)

The proof for the case $k \rightarrow 0+$ is similar.

Now, to prove the converse, assume that $G$ satisfies (5.10). Then for each $\phi \in M_{c+1, b+1}(I)$,

$$
\begin{aligned}
&\left|\left\langle\left(k^{\alpha} L(k)\right)^{-1} T(k t), \phi(t)\right\rangle\right| \\
&=\left|\left(k^{\alpha-1} L(k)\right)^{-1},\left\langle D_{t}^{N}\left(t^{N+1} \zeta_{c+1, b+1}(k t) G(k t)\right), \phi(t)\right\rangle\right| \\
&=\left|\left(k^{\alpha-1} L(k)\right)^{-1}(-1)^{N}\left\langle t^{N+1} \zeta_{c+1, b+1}(k t) G(k t), D^{N} \phi(t)\right\rangle\right| \\
&=\mid\left(k^{\alpha-1} L(k)\right)^{-1}(-1)^{N}\left[\int_{0}^{1 / k} t^{N+1} \zeta_{c+1, b+1}(k t) G(k t) D^{N} \phi(t) d t\right. \\
&\left.\quad+\int_{1 / k}^{\infty} t^{N+1} \zeta_{c+1, b+1}(k t) G(k t) D^{N} \phi(t) d t\right] \mid \\
& \leqq\left(k^{\alpha-1} L(k)\right)^{-1}\left[\int_{0}^{1 / k}|G(k t)| \frac{\zeta_{c+1, b+1}(k t)}{\zeta_{c+1, b+1}(t)}\left|t^{N+1} \zeta_{c+1, b+1}(t) D^{N} \phi(t)\right| d t\right.
\end{aligned}
$$




$$
\left.+\int_{1 / k}^{\infty}|G(k t)| \zeta_{c+1, b+1}(k t) / \zeta_{c+1, b+1}(t)\left|t^{N+1} \zeta_{c+1, b+1}(t) D^{N} \phi(t)\right| d t\right]
$$

Now, using (4.1), for large $k(>1)$, we have

$$
\begin{aligned}
\left|\left\langle\left(k^{\alpha} L(k)\right)^{-1}, \phi(t)\right\rangle\right| & \\
\leqq & \left(k^{\alpha-1} L(k)\right)^{-1} C_{1}\left[k^{-1} \int_{0}^{1}|G(u)| \zeta_{c+1, b+1}(u) / \zeta_{c+1, b+1}(u / k) d u\right. \\
& \left.+\int_{1 / k}^{\infty} C L(k t)(k t)^{\alpha-1} / \zeta_{c+1, b+1}(t) d t\right] \\
= & \left(k^{\alpha-1} L(k)\right)^{-1} C_{1}\left[k^{-1} \int_{0}^{1}|G(u)| k^{-c-1} d u+\frac{C^{\prime}}{k} \int_{1}^{\infty} L(u) u^{\alpha-1} / \zeta_{c+1, b+1}(u / k) d u\right] \\
= & \left(k^{\alpha-1} L(k)\right)^{-1} C_{1}\left[k^{-c-2} \int_{0}^{1}|G(u)| d u+\frac{C}{k}\left(k^{-c-1} \int_{1}^{k} L(u) u^{\alpha+c+\eta} u^{-\eta} d u\right.\right. \\
& \left.\left.+k^{-b-1} \int_{k}^{\infty} L(u) u^{\alpha+b+\eta^{\prime}} u^{-\eta^{\prime}} d u\right)\right] \\
= & C_{1} k^{-\alpha-c-1}(L(k))^{-1} \int_{0}^{1}|G(u)| d u \\
& +\left(k^{\alpha-1} L(k)\right)^{-1} C_{1} k^{-c-2} C^{\prime} L(k) k^{\alpha+c+\eta} \int_{0}^{k} u^{-\eta} d u \\
= & C_{1} k^{-\alpha-c-1}(L(k))^{-1} \int_{0}^{1}|G(u)| d u+C_{1} C^{1} /(1-\eta)+C_{1} C^{1} /\left(\eta^{\prime}-1\right) \\
& +\left(k^{\alpha-1} L(k)\right)^{-1} C_{1} k^{-b-2} C^{\prime} L(k) k^{\alpha+b+\eta^{\prime}} \int_{k}^{\infty} u^{-\eta^{\prime}} d u,(b y(5.1))
\end{aligned}
$$

which tends to $C_{1} C^{1}\left(1 /(1-\eta)+1 /\left(\eta^{\prime}-1\right)\right)$ as $k \rightarrow \infty$ for $\alpha+c+\eta>0, \quad \eta<1$ and $\alpha+b+\eta^{\prime}<0, \eta^{\prime}>1$.

Next for $k \rightarrow 0+$, using (5.11) we have 


$$
\begin{aligned}
\left|\left\langle\left(k^{\alpha} L(1 / k)\right)^{-1} T(k t), \phi(t)\right\rangle\right| & \\
\leqq & \left(k^{\alpha-1} L(1 / k)\right)^{-1} C_{1}\left[\int_{0}^{1 / k}|G(k t)| \zeta_{c+1, b+1}(k t) / \zeta_{c+1, b+1}(t) d t\right. \\
& \left.+\int_{1 / k}^{\infty}|G(k t)| \zeta_{c+1, b+1}(k t) / \zeta_{c+1, b+1}(t) d t\right] \\
\leqq & \left(k^{\alpha-1} L(1 / k)\right)^{-1} C_{1}\left[C \int_{0}^{1 / k} L(1 /(k t))(k t)^{\alpha-1} / \zeta_{c+1, b+1}(t) d t\right. \\
& \left.+\int_{1}^{\infty}|G(u)| \zeta_{c+1, b+1}(u) / \zeta_{c+1, b+1}(u / k) d u / k\right] \\
= & \left(k^{\alpha-1} L(1 / k)\right)^{-1} C_{1}\left[C\left(\int_{0}^{1} L(1 /(k t)) k^{\alpha-1} t^{\alpha+c} d t+\int_{1}^{1 / k} L(1 /(k t)) k^{\alpha-1} t^{\alpha+b} d t\right)\right. \\
& \left.+k^{-1} \int_{1}^{\infty}|G(u)| u^{-b-1} /(u / k)^{-b-1} d u\right] \\
= & \left(k^{\alpha-1} L(1 / k)\right)^{-1} C_{1}\left[C \left(k^{-c-2} \int_{1 / k}^{\infty} L(u) u^{-\alpha-c-2} d u\right.\right. \\
& +\left(k^{\alpha-1} L(1 / k)\right)^{-1} C_{1}\left[C^{\prime} k^{-c-2}(1 / k)^{-\alpha-c-\eta} L(1 / k) \int_{1 / k}^{\infty} u^{-2+\eta} d u\right. \\
& \left.+k^{-b-2} \int_{1}^{\infty}|G(u)| d u\right] \\
& \left.+k^{-b-2} \int_{1}^{1 / k} L(u) u^{-\alpha-b-2} d u+k^{-b-2} \int_{1}^{\infty}|G(u)| d u\right] \\
& +\left(k^{\alpha-1} L(1 / k)\right)^{-1} C_{1}\left[C k^{-c-2} \int_{1 / k}^{\infty} u^{-\alpha-c-\eta} L(u) u^{-2+\eta} d u\right. \\
& +\int_{1}^{-b-2} u^{-\alpha-b-\eta^{\prime}} L(u) u^{-2+\eta^{\prime}} d u \\
& \\
&
\end{aligned}
$$




$$
=C_{1} C^{1}\left(1 /(1-\eta)+1 /\left(\eta^{\prime}-1\right)\right)+C_{1}\left((1 / k)^{-\alpha-b-1} L(1 / k)\right)^{-1} \int_{1}^{\infty}|G(u)| d u
$$

which tends to $C_{1} C^{1}\left(1 /(1-\eta)+1 /\left(\eta^{\prime}-1\right)\right)$ as $k \rightarrow 0+$ provided that $\alpha+c+\eta>0, \eta<1$; $\alpha+b+\eta^{\prime}<0, \eta^{\prime}>1 ;$ and $\int_{1}^{\infty}|G(u)| d u<\infty$.

We shall use Theorem 5.2 in future investigations. However, the following form of definition of q.a.b. will be used in the sequel.

Definition 5.3. A generalized function $T$ in $M_{c, b}^{\prime}(\bar{I})$ has q.a.b. at infinity (at zero) of order $\alpha$ if the limit

$$
\lim _{k \rightarrow(0+)} k^{-\alpha} T(k t)=\gamma(t), \quad \gamma \in M_{c, b}^{\prime}
$$

exists in $M_{c, b}^{\prime}$ provided $\gamma(t) \neq 0$; if the limit in (5.13) exists we write $T(t) \sim \gamma(t)$ in $M_{c, b}^{\prime}$ as $t \rightarrow \infty(0+)$. If for any $\alpha$

$$
\lim _{k \rightarrow \infty(0+)} k^{-\alpha} T(k t)=0
$$

in $M_{c, b}^{\prime}$, we say that $T$ has order $-\infty$ as $t \rightarrow \infty(0+)$ and write $T \sim 0$ in $M_{c, b}^{\prime}$ as $t \rightarrow \infty(0+)$.

Theorem 5.4. Let $T$ be a generalized function belonging to $M_{c+1, b+1}^{\prime}(\bar{I})$ of the form (5.9). If $T$ has q.a.b. at $\infty$ (at zero) of order a according to the Definition 5.3, then there exists a complex constant $Q$ such that

$$
\lim _{t \rightarrow \infty(0+)} t^{-\alpha+1} \zeta_{c+1, b+1}(t) G(t)=Q
$$

Conversely, if $\alpha+b+1<0, \alpha+c+1>0$, and (5.14) holds, with the additional condition

$$
\int_{1}^{\infty}|G(t)| d t<\infty
$$

for q.a.b. at zero, then $T$ has q.a.b. at infinity (at zero) of order $\alpha$ in the sense of Definition 5.3 .

The proof is similar to that of Theorem 5.2.

The asymptotic behaviour of the generlized Wimp transform of elements $T \in M_{c+1, b+1}^{\prime}(\bar{I})$ of the form stated in (5.9) and having q.a.b. according to Definition 5.3 is now obtained by using the fact that such behaviour for $T$ implies (5.14) as given in Theorem 5.4.

Theorem 5.5. Let $T \in M_{c+1, b+1}^{\prime}(\bar{I})$ be of the form stated in (5.9) with the additional 
assumption that $G(t)$ is absolutely integrable over $(y, \infty)$ for all $y>0$. Let $T$ have q.a.b. at zero of order $\alpha$ according to Definition 5.3. Let $c \leqq 0$ and $-c<\alpha+1<-b$. For the constant $Q$ in (5.14) and order $N$ of $T$ in (5.9) we have

$$
\lim _{\substack{|s| \rightarrow \infty \\ s \in Q_{k}}}\left(\Gamma(v-s-\alpha) \Gamma(v+s-\alpha) H_{1}(\alpha)\right)^{-1} F[T ; s]=(-1)^{N} Q
$$

where $Q_{k} \subset \mathbb{C}^{U} \cap\left\{s:\left|s_{1}\right|<v_{1}-\alpha-1\right\}$ and

$$
\mathscr{H}_{1}(\alpha)=\frac{\prod_{j=1}^{m} \Gamma\left(b_{j}+\alpha+1\right) \prod_{j=1}^{n} \Gamma\left(-a_{j}-\alpha\right) \Gamma(-\alpha)}{\prod_{j=m+1}^{q} \Gamma\left(-b_{j}-\alpha\right) \prod_{j=n+1}^{p} \Gamma\left(a_{j}+\alpha+1\right) \Gamma(-N-\alpha)}
$$

Proof. We have

$$
\begin{aligned}
F[T ; s] & =\left\langle D_{t}^{N}\left(\zeta_{c+1, b+1}(t) t^{N+1} G(t)\right), G(t, s)\right\rangle \\
& =(-1)^{N}\left\langle\zeta_{c+1, b+1}(t) t^{N+1} G(t), D_{t}^{N} G(t, s)\right\rangle \\
& =(-1)^{N} \int_{0}^{\infty} \zeta_{c+1, b+1}(t) t G(t) G_{p+3, q+1}^{m, n+3}\left(\left.t\right|_{b_{q}, N} ^{0,1-v+s, 1-v-s, a_{p}}\right) d t .
\end{aligned}
$$

By the conclusion (5.14) of Theorem 5.4 there exists a complex constant $Q$ such that as functions

$$
t \zeta_{c+1, b+1}(t) G(t) \sim Q t^{\alpha} \quad \text { as } t \rightarrow 0+
$$

From the integrability hypothesis on $G(t),\left(t^{b}\left(t \zeta_{c+1, b+1}(t) G(t)\right)\right.$ is absolutely integrable on $(y, \infty)$ for all $y>0$. Further,

$$
t^{-\alpha}\left(t \zeta_{c+1, b+1}(t) G(t)\right)=t^{-a+1} \zeta_{c+1, b+1}(t) G(t)
$$

is bounded on $0<t \leqq y$ for all $y>0$ because of (5.14) as $t \rightarrow 0+$ and the continuity of $G(t)$. Recalling that $b=\max (d,-1)$ we can apply the proof of Theorem 3.1 to the function $\left(t \zeta_{c+1, b+1}(t) G(t)\right)$ with $\eta=\alpha$ and get

$$
\begin{aligned}
& \lim _{\substack{|s| \rightarrow \infty \\
s \in Q_{k}}}(\Gamma(v-s-\eta) \Gamma(v+s-\eta))^{-1} \int_{0}^{\infty} t \zeta_{c+1, b+1}(t) G(t) \\
& \quad \times G_{p+3, q+1}^{m, n+3}\left(\left.t\right|_{b_{q}, N} ^{0,1-v+s, 1-v-s, a_{p}}\right) d t \\
& \quad=Q \mathscr{H}_{1}(\alpha) .
\end{aligned}
$$


Hence (5.17) and (5.18) yield (5.15).

Theorem 5.6. Let $T \in M_{c+1, b+1}^{\prime}(I)$ be of the form (5.9) and have q.a.b. at infinity of order $\alpha$ according to Definition 5.3. Let $c \leqq 0$. Then for the constant $Q$ in (5.14) and order $N$ of $T$ in (5.9) we have

$$
\lim _{\substack{|s| \rightarrow \infty \\ s \in Q_{k}}}\left(\Gamma(v-s-\alpha) \Gamma(v+s-\alpha) H_{1}(\alpha)\right)^{-1} F[T ; s]=(-1)^{N} Q
$$

where $Q_{k} \subset \mathbb{C}^{U} \cap\left\{s:\left|s_{1}\right|<v_{1}-\alpha-1\right\}$ and $\mathscr{H}_{1}(\alpha)$ is given by (5.16).

Proof. As in the proof of Theorem 5.3 we obtain (5.17) in this case. By the conclusion (5.14) of Theorem 5.4 there exists a complex constant $Q$ such that as functions

$$
t \zeta_{c+1, b+1}(t) G(t) \sim Q t^{\alpha} \quad \text { as } \quad t \rightarrow \infty
$$

Further, $t^{c}\left(t \zeta_{c+1, b+1}(t) G(t)\right)$ is absolutely integrable over $(0, y)$ (for all $y>0$; and $\left(t^{-\alpha}\left(t \zeta_{c+1, b+1}(t) G(t)\right)\right.$ is bounded on $y \leqq t<\infty$ for all $y>0$ because of (5.14) for $t \rightarrow \infty$ and the continuity of $G(t)$. Since $b=\max (d,-1)$, the proof of Theorem 3.2 applied to $\left(t \zeta_{c+1, b+1}(t) G(t)\right)$ with $\eta=\alpha$ yields (5.19) from the identity (5.17) in a similar way to the proof of Theorem 5.3. This completes the proof.

Now we give an example of a distribution in $M_{c+1, b+1}^{\prime}(\bar{I})$ which possesses q.a.b. at infinity and at zero.

Let

$$
T=D_{t}\left(\zeta_{c+1, b+1}(t) \zeta_{\sigma, \rho}(t) t^{2} I(t)\right)
$$

where $I(t)$ is the characteristic function of $[0, \infty)$. Now, if

(i) $\rho>1$ and $b+\rho-c<\sigma<1$, the $T \in M_{c+1, b+1}^{\prime}$ has q.a.b. at infinity of order $\alpha=-b-\rho$ according to Definition 5.1 with

$$
\gamma(t)=(1-b-\rho) t^{-b-\rho} I(t) \in M_{c+1, b+1}^{\prime}
$$

and if

(ii) $\sigma<1$ and $c+\sigma-b>\rho>1$, then $T \in M_{c+1, b+1}^{\prime}$ has q.a.b. at zero of order $\alpha=$ $-c-\sigma$ with

$$
\gamma(t)=(1-c-\sigma) t^{-c-\sigma} I(t) \in M_{c+1, b+1}^{\prime}
$$

Acknowledgement. The work was supported by U.G.C. Grant No. P-01/214. 


\section{REFERENCES}

1. R. Bojanic and J. Karamata, On slowly varying functions and asymptotic relations (MRC Technical Summary Report 432, October 1973, Madison, Wisconsin).

2. R. D. Carmichael and E. O. Milton, Abelian theorems for the distributional Stieltjes transform, J. Math. Anal. Appl. 72 (1979), 195-205.

3. R. D. Carmichael and R. S. Pathak, Asymptotic behaviour of the $H$-transform in the complex domains, Math. Proc. Cambridge Philos. Soc. 102 (1987), 533-552.

4. Ju. N. Drozżınov and B. I. Zavialov, The quasi-asymptotics of generalized functions and tauberian theorems in the complex domain, Math. USSR Sbornik 31 (1977) 329-345.

5. I. M. Gel'fand and G. E. Shilov, Generalized functions, vol. I (Academic Press, New York, 1964).

6. D. S. Jones, Generalized transforms and their asymptotic behaviour, Philos. Trans. Roy. Soc. London Ser. A 265 (1969), 1-43.

7. V. G. Joshi and R. K. SAXENA, Abelian theorems for distributional $H$-transform, Math. Ann. 256 (1981), 311-321.

8. J. Lavoine and O. P. Misra, Théorèmes abéliens pour la transformation de Stieltjes des distributions, C.R. Acad. Sci. Paris Serie A 279 (1974), 99-102.

9. Y. L. Luke, The Special Functions and Their Approximations (Academic Press, New York, 1969).

10. R. S. Pathak, Abelian theorems for the $G$-transformation, J. Indian Math. Soc. 45 (1981), 243-249.

11. L. Schwartz, Théorie des Distributions (Hermann, Paris, 1966).

12. B. Stankovic, Abelian and tauberian theorems for Stieltjes transforms of distributions, Russian Math. Surveys 40:4 (1985), 99-113.

13. A. TAKać, A note on distributional Stieltjes transformation, Math. Proc. Cambridge Philos. Soc. 94 (1983), 523-527.

14. F. Treves, Topological vector spaces, Distributions and Kernels (Academic Press, New York, 1966).

15. J. WIMP, A class of integral transforms, Proc. Edinburgh Math. Soc. 14 (1964), 33-40.

16. R. Wonc, Asymptotic expansions of the Kontorovich-Lebedev Transform, Applicable Anal. 12 (1981), 161-172.

17. A. H. Zemanian, Some abelian theorems for the distributional Hankel and $K$ transformations, SIAM J. Appl. Math. 14 (1966), 1255-1265.

18. A. H. Zemanian, Generalized Integral Transformations (Interscience Publishers, New York, 1968).

Department of Mathematics

Banaras Hindu University

VARANASI 221005

INDIA 